\title{
RHABDOMYOSARCOMA OF THE LEFT ATRIUM
}

\author{
BY
}

\section{W. N. DUNNET, C. SYMONS, AND CYNTHIA STEPHENSON}

From the Departments of Infectious Diseases, Cardiology, and Pathology, Royal Free Hospital

Rhabdomyosarcoma of the heart is exceedingly rare, 24 cases only having been recorded. This report describes a further case, of particular interest because of the unusual clinical presentation.

\section{Case Report}

A woman, aged 60 years, was admitted to the Royal Free Hospital with a history of severe diarrhœa and vomiting, with blood in the stools for one week. For two years she had been in poor health with episodes of giddiness, blurred vision, loss of consciousness, and numerous falls; six months previously she had been admitted to another hospital with "pyrexia of unknown origin". Relevant signs in the cardiovascular system at that time were persistent systolic and transient diastolic murmurs at the apex. Screening of the chest revealed left atrial enlargement, and the X-ray film (Fig. 1) showed an opacity within the heart shadow

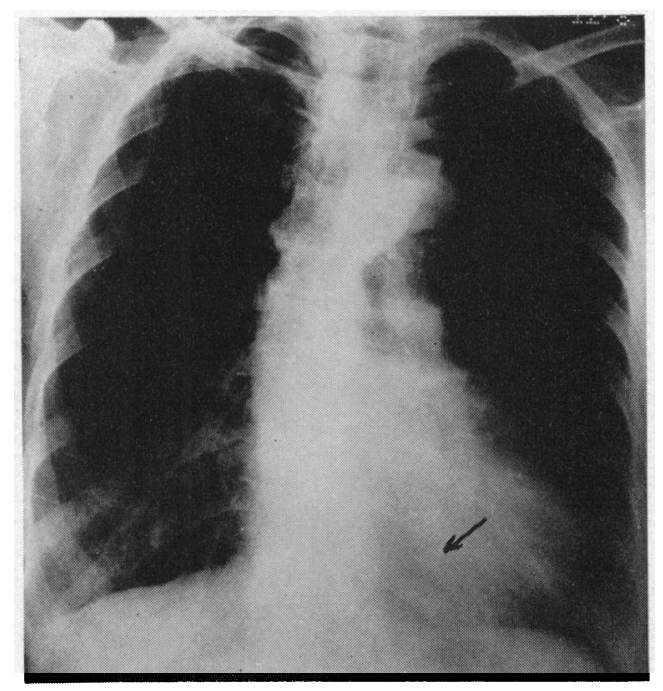

FIG. 1.-X-ray film showing abnormal opacity within the left heart border caused by the rhabdomyosarcoma. The arrow indicates the inferior portion of the tumour projecting through the mitral valve.

which, considered in retrospect when the diagnosis had been established at autopsy, was undoubtedly due to the rhabdomyosarcoma. A provisional diagnosis of subacute bacterial endocarditis was made, although repeated blood cultures had been negative, and prolonged courses of several antibiotics had been given without controlling the pyrexia. She had become progressively more anæmic and had been given blood transfusions. Following discharge from hospital she had been virtually bed-ridden, being unable to stand because of faintness in the erect position and weakness in the legs. 
Other points in the past history were that at fifty years of age subtotal hysterectomy for fibroids and appendicectomy had been performed. The following year she had been treated for syphilis, and three years later tabes dorsalis had been diagnosed.

Clinical Findings. On admission the patient was gravely ill, emaciated, and severely dehydrated; the temperature was subnormal, and peripheral circulatory failure was present as shown by a poor volume pulse, cold extremities, and low blood pressure (80/60). The heart was enlarged, the apex beat being in the sixth intercostal space and half an inch outside the midclavicular line; the heart sounds were of poor quality and the rhythm was irregular, due to atrial fibrillation. There was an apical systolic murmur, but no diastolic murmur was heard then or subsequently. The liver was easily palpable, and there was neurological evidence of tabes dorsalis.

A diagnosis of acute gastro-enteritis with peripheral circulatory failure was made.

Laboratory Investigations. Hb. 11.4 g. $(77 \%)$. W.B.C. 16,000, with a neutrophil leucocytosis. Platelets 235,000 per cu. mm. E.S.R. $56 \mathrm{~mm}$. (Westergren). Blood urea $70 \mathrm{mg}$. $/ 100 \mathrm{ml}$. Serum potassium $2.8 \mathrm{mEq} . / 1$. Serum sodium $128 \mathrm{mEq}$./1. Serum chloride $90 \mathrm{mEq} . /$. Total serum protein $4 \cdot 1$ g. $/ 100 \mathrm{ml}$., albumin $1.7 \mathrm{~g} . / \mathrm{ml}$., globulin $2.4 \mathrm{~g} . / \mathrm{ml}$.; the serum electrophoretic pattern revealed a decrease in the globulin fraction and slight increase in the $\alpha_{2}$ globulin. The urine contained albumin, but was otherwise normal. The Kahn test was positive, the W.R. and G.C.F.T. were negative. Culture of fæces showed no pathogenic organisms. Increased shadowing at both bases was demonstrated by portable chest X-ray, but the changes seen in Fig. 1 were not evident due to the poor quality of the portable film. The electrocardiograms revealed uncontrolled atrial fibrillation and changes consistent with hypokalæmia.

Progress. Measures to combat circulatory failure were instituted. The fluid and electrolyte imbalance was corrected, and digoxin, $0.5 \mathrm{mg}$., given intravenously, followed by $0.25 \mathrm{mg}$. orally every eight hours. Penicillin and streptomycin were administered parenterally. As the blood pressure did not improve an infusion of nor-adrenaline was given together with hydrocortisone, $100 \mathrm{mg}$. eight-hourly. With this treatment the blood pressure rose to $125 / 80$ and the clinical condition was fairly satisfactory until the sixth day after admission when it suddenly deteriorated, and the patient died twelve hours later. She had been afebrile throughout the illness.

Necropsy. 1. Rhabdomyosarcoma of heart. 2. Metastases (liver, bone marrow, ileum, brain). 3. Mitral obstruction. 4. Endocardial thrombi. 5. Chronic atrophic emphysema. 6. Right ventricular failure. 7. Amyloidosis (spleen, ileum, adrenal, pancreas, kidneys). 8. Chronic pyelonephritis (mild). 9. Demyelination of the posterior columns (mild). 10. Fatty liver. 11. Hæmorrhoids. 12. Atheroma (mild).

Relevant findings were confined to the heart, gastro-intestinal tract, liver, vertebral bone marrow, and brain. The heart weighed 276 g., the right side being moderately dilated but not hypertrophied. On opening the left side of the heart a firm polypoid mass was revealed: this projected through, and almost completely obstructed, the mitral orifice (Fig. 2). This mass arose from a wide base on the posterior wall of the left atrium and measured $9.5 \times 2.5 \times 2.5 \mathrm{~cm}$. The external surface was irregular, mottled yellow and red, and covered by fibrin thrombus. The cut surface was yellow-grey and showed a poorly defined lobular pattern. There were numerous small fibrin deposits and petechial hæmorrhages in the endocardium of the atrium and along the base of the mitral valve. Small metastatic tumour deposits were present in the liver, small intestine, vertebral bone marrow, and the left frontal lobe of the brain. The alimentary tract showed no obvious lesion to account for the gastro-enteritis.

The cause of death was cardiac failure due to mitral valve obstruction by a neoplasm of the left atrium.

Histology. The heart tumour was a pleomorphic sarcoma showing a tendency to a myomatous pattern in some areas. The predominant cells were long, slender straps with central nuclei, which showed considerable variation in size, shape, and degree of hyperchromatism. With phosphotungstic acid and hæmatoxylin (P.T.A.H.) staining, these cells showed longitudinal fibrils, and a few cross striations were demonstrated. Large multinucleate cells with eosinophilic cytoplasm were scattered among the anaplastic sarcoma cells. On this evidence the tumour was diagnosed as a rhabdomyosarcoma. The metastatic deposits had the same histological characters as the heart tumour. Tumour cells were present in the endocardium underlying the fibrin thrombi.

Extensive amyloid deposits were found in the spleen, small intestine, pancreas, and adrenals. Small deposits were also found in the kidneys which showed a mild degree of chronic pyelonephritis. Sections of the spinal cord taken from the cervical, thoracic, and lumbar areas showed mild demyelination in the posterior columns.

\section{Discussion}

Whorton (1949) reviewed 100 cases of primary malignant tumours of the heart, of which 9 were rhabdomyosarcoma. In a further review, Cruz and Ana (1956) reported several other cases of rhabdomyosarcoma, bringing the total number to 20, without the case of Friedlander et al. (1950) which was described as a myosarcoma associated with malignant carcinoids and pancreatic heterotopia. The report of another 


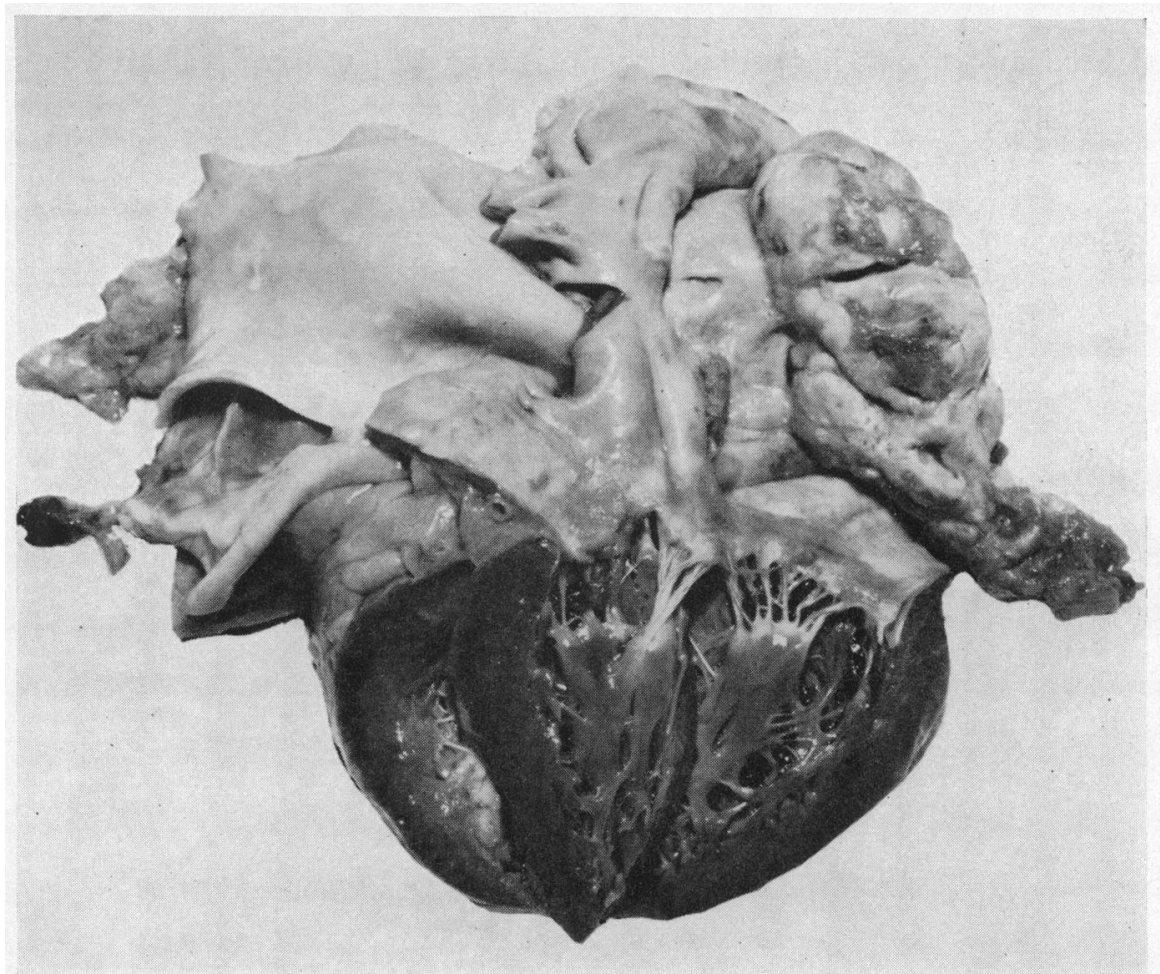

FIG. 2.-Rhabdomyosarcoma attached to the wall of the left atrium, with a tongue-like downward projection passing through the mitral valve into the left ventricle. The lower part of the tumour has been displaced laterally to show the mitral leaflets.

case from Poland in 1955 is not available in this country for study. Manson and Rindskopf (1956) reported a case in a girl, aged 12, with extensive myocardial involvement. Pascuzzi et al. (1957) in a review of rhabdomyosarcoma added one further case, a woman aged 25 , in whom the diagnosis was made in life by angiocardiography and pericardial biopsy.

The clinical presentation of a primary intracardiac tumour obstructing the mitral valve has been well described, and Fawcett and Ward (1939) have summarized the symptoms and signs as follows: signs suggestive of mitral stenosis with congestive cardiac failure and changing cardiac murmurs, fainting attacks and loss of consciousness with the patient erect, sudden and unexpected death, relentless progress of heart failure in spite of adequate rest and digitalization, abnormal X-ray shadows, cardiac irregularities, and paroxysmal dyspnœa. These abnormalities were particularly described in relation to cardiac myxoma but would equally apply to any tumour obstructing the mitral valve. The case described here presented only four of the findings enumerated, namely, fainting attacks, cardiac irregularity, a variable cardiac murmur, and an abnormal X-ray.

Von Reis (1949), describing the clinical aspects of 30 cases of endocardial myxoma, reported that endocarditis was a frequent misdiagnosis. The gastro-enteritis was most probably a terminal complication resulting from the poor general condition of the patient.

Other important points in the history were fainting attacks and loss of consciousness, due to the tumour filling the mitral orifice during cardiac contraction (Jones and Julian, 1955). In retrospect, careful scrutiny of one of the chest X-rays suggested that the diagnosis of intracardiac tumour could have been made, particularly if fluoroscopy and tomography had been performed. The solid nature of the rhabdomyosarcoma was particularly striking at autopsy.

The amyloid deposits were unrelated to the cardiac condition and were probably secondary to the syphilitic infection. 


\section{Summary}

A case of rhabdomyosarcoma is described in a woman, aged 60, who had suffered from unexplained fainting attacks with loss of consciousness for two years. A syphilitic infection had been diagnosed at the age of 51 years, and she had developed tabes dorsalis three years later. She presented terminally as a case of acute gastro-enteritis and peripheral circulatory failure.

Post mortem, the tumour almost completely obstructed the mitral valve. Extensive amyloid deposits were found in the spleen, small intestine, pancreas, and adrenal glands.

A previous chest X-ray, examined in retrospect when the pathological diagnosis was known, revealed an opacity within the heart shadow that was almost certainly due to the rhabdomyosarcoma.

We are greatly indebted to Dr. W. Gunn and Dr. A. M. Ramsay for their encouragement and advice in preparing this paper, and to Professor Kenneth R. Hill for his valuable criticism. We would also like to thank Dr. R. Finlayson for his help with the histology and all those in the Photographic Department of the Royal Free Hospital for their willing co-operation. It is a pleasure to mention Miss Beacock who was most helpful with the references.

\section{References}

Cruz, J. Z. S., and Ana, D. S. S. (1956). J. Phil. med. Ass., 32, 251.

Fawcett, R. E. M., and Ward, E. M. (1939). Brit. Heart J., 1, 249.

Friedlander, S., Hein, G. E., and Siemens, J. C. (1950). Circulation, 1, 797.

Jones, G. P., and Julian, D. G. (1955). Brit. med. J., 2, 361.

Malecka-Dymnicka, S., and Zawrockawrzolek, T. (1955). Polskie Arch. med. wewn., 25, 987.

Manson, G., and Rindskopf, W. (1956). J. Pediat., 48, 202.

Pascuzzi, C. A., Parkin, T. W., Bruwer, A. J., and Edwards, J. E. (1957). Proc. Mayo Clin., $32,30$.

von Reis, G. (1949). Acta med. scand., 133, 214.

Whorton, C. M. (1949). Cancer, N.Y., 2, 245. 